\title{
Highlighting photonics: looking into the next decade
}

\author{
Zhigang Chen ${ }^{1,2^{*}}$ (1) and Mordechai Segev ${ }^{3}$
}

\begin{abstract}
Let there be light-to change the world we want to be! Over the past several decades, and ever since the birth of the first laser, mankind has witnessed the development of the science of light, as light-based technologies have revolutionarily changed our lives. Needless to say, photonics has now penetrated into many aspects of science and technology, turning into an important and dynamically changing field of increasing interdisciplinary interest. In this inaugural issue of elight, we highlight a few emerging trends in photonics that we think are likely to have major impact at least in the upcoming decade, spanning from integrated quantum photonics and quantum computing, through topological/non-Hermitian photonics and topological insulator lasers, to Al-empowered nanophotonics and photonic machine learning. This Perspective is by no means an attempt to summarize all the latest advances in photonics, yet we wish our subjective vision could fuel inspiration and foster excitement in scientific research especially for young researchers who love the science of light.
\end{abstract}

Keywords: Integrated quantum photonics, Photonic quantum computing, Topological photonics, Non-Hermitian photonics, Topological insulator lasers, Al-empowered nanophotonics, Photonic machine learning

\section{Introduction}

Photonics plays a crucial role in our daily lives, as the science of light and associated technological development is turning into an economic and social imperative in the twenty-first century. To mention just one example, what make today's internet possible are the inventions of optical fiber communication, the distributed feed-back lasers, and the fiber amplifiers. Equally important, the science of light has led to numerous fundamental discoveries. Perhaps, one of the most profound is the first experiments on quantum entanglement $[1,2]$ that started the second quantum revolution, performed strictly with photons. Many other discoveries were driven by progress in laser science, ranging from for example experimental realization of Bose-Einstein condensates [3], demonstration of quantum simulators [4], high harmonic generation

\footnotetext{
*Correspondence: zgchen@nankai.edu.cn

${ }^{1}$ The MOE Key Laboratory of Weak-Light Nonlinear Photonics, TEDA Applied Physics Institute and School of Physics, Nankai University, Tianjin 300457, China

Full list of author information is available at the end of the article
}

and attosecond science [5-7], to super-resolution laserdriven microscopy [8]. Indeed, for the past 25 years or so, the largest number of Nobel Prizes in physics is in the general area of light science.

Here, we do not dwell into the past, but rather provide a glimpse into a few emerging trends in photonics that we think are important and will continue to advance in the next decade.

\section{Integrated quantum photonics and photonic quantum computing}

Quantum photonics, a field that has advanced rapidly in the past decade, deals broadly with fundamental science and technology where quantum effects play the essential role. Loosely speaking, quantum photonics focuses on the applications based on quantized light-matter interaction, where it often involves manipulation and active control of light and matter at the quantum level, either for single photons, entangled pairs of photons and/or atoms, or their cluster states. Although research into quantum photonics has a history almost as venerable as that of the 
laser, only in recent decades efforts have become more focused on the practical applications of photonic quantum technologies $[9,10]$. Quantum technologies are expected to have the potential to play important roles in many applications such as quantum metrology, quantum information processing, quantum thermometry, and quantum bio-marking and sensing. An exemplary success is in the area of quantum cryptography which has been highly tested and advocated for secure communication, from ground-based quantum key distribution to satellite quantum communications $[11,12]$. Perhaps, one of the most important quantum technologies is the holy grail of making a real quantum computer: real in the sense that it is fault-tolerant and can solve computation problems that classical computers cannot, even within a span of a century. Quantum computers are now being extensively explored, with important progress in several platforms such as superconductors, ion traps, neutral atoms, and particularly silicon photonics circuitries, although there is still a long way to go [13]. Here, integrated quantum photonics (IQP) plays a major role and might win the competition for realizing a real quantum computer.

Some of recent progresses in the IQP area were reviewed in a special focus issue of Nature Photonics [14, 15], where one can find an introduction to IQP circuits and a general overview of the advances in IQP technologies along with demonstrated applications, as well as the progress of the hybrid IQP devices (with assembled optical components made from different material systems) and the roadmap for realizing future advanced large-scale hybrid quantum devices. Towards the goal of realization of on-chip quantum photonics, silicon-based materials have been actively explored for IQP due to their compatibility with conventional CMOS fabrication processes. For example, Peyskens and co-workers [16] recently demonstrated the integration of single photon emitters in twodimensional (2D) layered materials with a silicon nitride photonic chip, promising for single photon generation and extraction in scalable quantum photonic circuits. Paesani and co-workers [17] developed a novel nonlinear optical technique to generate near-ideal integrated photon sources in silicon with the potential to deliver large-scale quantum photonics on a single chip. Lukin and co-workers [18] demonstrated single-photon emission and efficient optical frequency conversion from an IQP chip based on silicon carbide. Very recently, Fadaly and co-workers [19] demonstrated efficient light emission from hexagonal silicon-germanium alloys similar to that from direct-bandgap semiconductors. All these light sources, aiming towards building blocks for quantum photonic circuits, are based on CMOS-compatible processes, making them particularly attractive for IQP and quantum computing in a single and monolithic platform.
In addition to those, the past decade has witnessed major progress on quantum dot sources of entangled photon states. In fact, already in 2006, Gershoni's group demonstrated entangled photon states "on demand" from a semiconductor quantum dot [20], and more recently photonic cluster states on demand [21]. Importantly, such quantum dot sources are designed to emit only the entangled states they were designed for, unlike all other sources (e.g., from spontaneous parametric downconversion and four wave-mixing) that emit statistical quantum light. For these reasons, quantum dot sources are considered as good candidates for quantum circuitry [22].

The progress on single photon sources and sources for entangled photon states and cluster states suggests the possibility of photonic quantum computing. Essentially, most of the routes to quantum computing include photonic settings in one form or another. As with classical computing, quantum computing typically requires logic gates that can be switched by a single quantum state. For example, a gating device that would be switched by a single photon, such that the first photon state passes through and switches the gate to reflect the second photon state [23]. In other words, almost all quantum computing schemes require a "two-QBit gate" that includes a nonlinear interaction driven by a single photon. This poses major challenges, because the interaction between a single photon and an atom, a molecule, or a quantum dot, is generally very weak. This is why single-photon nonlinear optics requires extreme conditions such as ultra-high-Q resonators and the like [24]. The implications of the difficulty in making a 2-QBit quantum gate are far reaching. They basically imply that, at present, there are only a handful of technologies that are widely considered as scalable platforms for quantum computing: superconductors (where the research and development is led by giant companies like IBM and Google, and is beyond the scope of this perspective article), ion traps [25-27] and the so-called "one-way photonic quantum computing" $[28,29]$ based on silicon photonic circuitry currently led by companies like PsiQuantum (https:// psiquantum.com) and Xanadu (https://xanadu.ai), as well as the major efforts led by Pan, Lu and coworkers in China (Fig. 1a) [30]. All other technologies for quantum computing are uncertain in their ability to scale up for a real quantum computer-that can actually do something useful (such as breaking codes), although some of them have already demonstrated impressive abilities in the closely related area of photonic programmable quantum simulators, for example, the 51-atom quantum simulator [31] and the diamond quantum nanophotonics [32]. In our opinion, photonics is currently the only viable platform for achieving a fault-tolerant quantum 

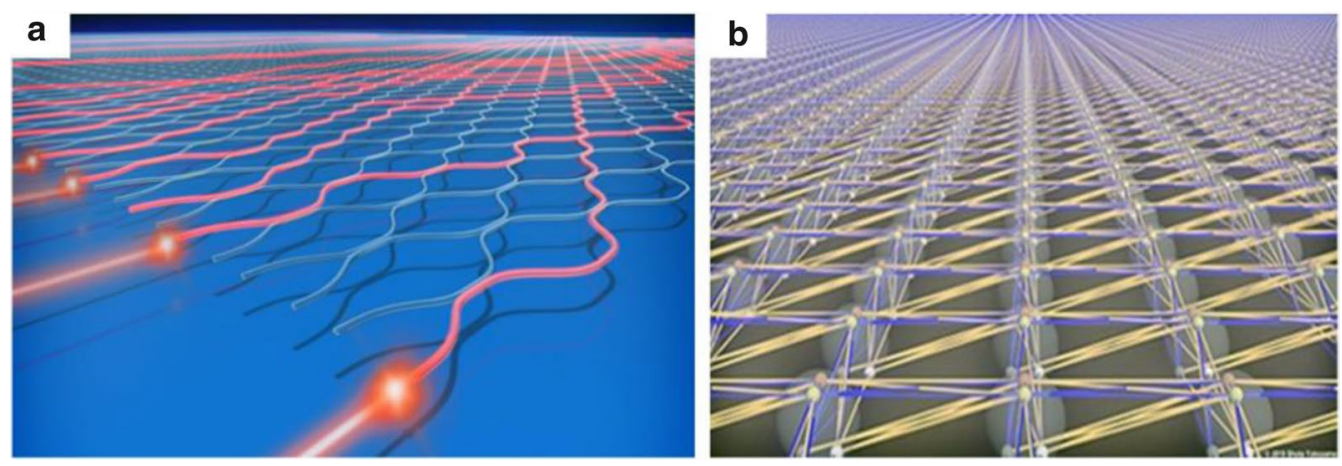

Fig. 1 a Artwork illustrating the boson sampling experiment to achieve photonic quantum supremacy, as established in a quantum computer called "Jiuzhang" [30] [Image credit:Yihan Luo/ScienceNews]. b Artwork illustrating the entanglement structure of a large-scale quantum processor made of light, highlighting the work from two research teams on the development of entangled 2D cluster states promising for "one-way" quantum computing $[35,36]$ [Image credit: Shota Yokoyama]

computer in near future. The platform is characterized by low error rates (estimated to be lower than $10^{-5}$ ) which are several orders of magnitude below those characterizing platforms that use the matter-based qubits. This comes with the price of employing non-deterministic linear optical gates, which give an overhead on the number of photons used to generate a photon that is actually used in the computation itself. However, this overhead can be seen to be favorable to the immense overhead of performing error correction or distillation to bring matter qubits to a similar error level. In fact, the stupendous amount of resources required for fault tolerant operation of quantum algorithms (estimated to be on the order of $10^{15}-10^{17}$ qubits multiplied by time-steps) highlights the great advantage of using a platform based on silicon photonics, as CMOS is the only currently available technology capable of supporting devices on very large scales (larger than $10^{7}$ components).

Let us elaborate on the photonic quantum computing technology. The entire approach of this technology is not based on quantum gates at all, but rather this "oneway quantum computing" (or "measurement-based quantum computing") implements in a completely different way, as first proposed about two decades ago [28] and demonstrated using the polarization states of photons [33]. Unlike all other techniques studied or proposed for quantum computing-which are based on single-qubit and entangling gates (e.g., two-qubit gates) chosen according to the implemented algorithm, photonic quantum computing does not rely on any physical gates during the computation. The photonic quantum computing scheme begins with the preparation of a standard entangled resource state which is independent of the algorithm implemented (e.g., a cluster state). The photons comprising this quantum state are launched into a predesigned integrated photonic circuit. The computation itself is performed by a continuous cycle of single photon coincidence measurement and dynamical updates to the connectivity of the channels of the circuit (via electro-optic switches or alike) based on the measurement result and the specific algorithm implemented. Thus, this scheme of computing does not require any logic gate whatsoever: the role of gating (a nonlinear switching unit) is played by the measurement itself. It is "one-way" because the resource cluster state is destroyed by the measurements, and it is advantageous because the cluster-state quantum computation does not a priori rely on any gate (quantum or classical). The great advantage of this technique is the ability to create an entangled state of a large number of photons, which is at the same time sufficiently free of errors and large enough to allow the use of topological error correction codes and therefore to operate in a fault tolerant manner. Constructing a device with a comparable number of physical qubits and fidelity which can allow the use of error correcting codes does not seem possible at least in the near future using any other technology.

There are many research groups worldwide are now working actively on quantum cluster states. For example, Reimer et al. [34] experimentally realized threelevel, four-partite cluster states formed by two photons in order to perform proof-of-concept high-dimensional one-way quantum operations with integrated photonic chips. Recently, Asavanant et al. and Larsen et al. [35, 36] reported the generation of large-scale cluster states of quantum continuous variables by use of a combination of quantum "squeezed light" to create massive, quantum-entangled 2D cluster states of light (Fig. 1b). It is yet unknown if this approach can achieve the first 
real quantum computer, although it is already clear that photonic one-way quantum computing has some major advantages over other approaches.

As gigantic companies like IBM, Google and Microsoft are racing to build general-purpose quantum computers, joined by many others in China and worldwide with increased investment, it seems promising that quantum computing will no longer be just a dream or science fiction. We believe that it is only a matter of time, and that quantum computers will change our world. Clearly, the science of light and associated photonic technologies will play a major role in this quantum arena.

\section{Topological/non-hermitian photonics and topological insulator lasers}

The theoretical discovery of topological phases of matter, manifested in many intriguing phenomena such as the quantum Hall effect and topological insulators, led to the 2016 Nobel Prize in physics. In a nut shell, topological insulators are materials that are insulators in the bulk but perfect conductors on their edges. The edge current exhibits the so-called "topologically protected transport": any wavepacket comprising superposition of edge states experiences unidirectional transport, robust against defects and disorder so long as the variation in the potential is smaller than the topological bandgap. The first discovered topological insulator was the quantum Hall effect (1985 Nobel Prize in physics), which relied on coherent transport of electrons subject to strong magnetic fields, dictating unidirectional topologically-protected transport (via the Lorentz force) of edge states in the topological bandgap. Modern topological insulators rely on other effects, such as spin-orbit interaction, temporal modulation of the underlying potential, and more. In its original domain of electronic systems, topological insulators rely on fermionic effects. However, already 15 years ago, researchers started to wonder whether the basic concepts of topological insulators are unique to fermions or they could be extended to bosonic systems. The understanding was that, if the ideas can indeed be generalized to bosons, then the underlying concepts are universal-apply also to all classical wave systems in nature. The first suggestion for a bosonic topological system was made by Haldane (one of the 2016 Nobel laureates) and Raghu in 2008 [37], who realized that breaking time-reversal symmetry in a photonic crystal could lead to electromagnetic topological insulators. The natural candidate to do that was using the magneto-optic effects in a photonic crystal. Indeed, within a year, the group of Soljačić demonstrated the idea in a photonic crystal made of gyro-optic material [38]. However, unlike other situations, for topological insulators the size actually matters: the topological protection of transport directly relies on the size of the bandgap. Alas, all magneto-optic effects are fundamentally very weak at frequencies of terahertz and higher, hence the protection at optical frequencies would be virtually non-existent because that slight disorder would close the topological gap. Accordingly, the pioneering work in gyro-optic photonic crystal [38] was performed with microwaves, where the magneto-optic effects can be strong. The goal of making photonic topological insulators at optical frequencies remained a major challenge for another few years, with many suggestions relying on various effects that do not rely on magnetic fields. Eventually, in 2013, the first photonic topological insulators were demonstrated: the Floquet photonic topological insulator relying on modulation [39] (based on an idea suggested in solid state physics [40]), and an aperiodic arrangement of coupled resonators emulating the integer quantum Hall effect [41]. Within a few years, numerous other mechanisms for photonic topological insulators were proposed and demonstrated in experiments, ranging from bianisotropic metamaterials $[42,43]$ and systems relying on the valley-Hall effects [44], to topological interfaces between expanded and shrunk honeycomb lattices [45] and periodic networks of strongly coupled resonators $[46,47]$.

Importantly, topological insulators do not necessarily need to rely on periodic structures in real-space; rather, they can rely on a ladder of equally-separated energy states, such as cavity modes $[48,49]$ or Bloch modes in a waveguide array judiciously designed as the so-called $J_{x}$ lattice [50]. Such topological insulators employing nonspatial dimensions are commonly referred to as "synthetic-space topological insulators", first demonstrated in cold atom systems [51, 52]. In photonics, the first synthetic-space topological insulator was demonstrated only recently in 2019 [50]. One of the interesting features of such synthetic-space systems is that they can exhibit a variety of phenomena that cannot be observed in real-space systems, for example, multi-dimensional topological dynamics [50], long-range connectivity, nonlocal disorder, and topological transport in the bulk [50, 53-55]. The necessity of a topological bandgap seems to imply that topological insulators must rely on a periodic structure (in real space or in synthetic dimensions). But, in fact, having a periodic potential is not at all required for having a topological insulator. It is indeed necessary to have a gap, which has to be topological. But, as was recently shown, modulation can readily induce a topological gap in structures lacking periodicity altogether, such as quasicrystals [56] or even fractal structures [57].

Research activities on topological photonics has grown tremendously in the past decade, with much progress made in implementing topological phases of light using different platforms such as metamaterials, surface plasmons, exciton-polaritons [58] photonic crystals, 
waveguide lattices and coupled cavities, as covered in quite a few review papers [59-63]. New findings and discoveries emerge popularly in topologically protected edge states and topological insulators, topological phases in synthetic dimensions, higher-order topological insulators, non-Abelian topological photonics, nonlinear and quantum topological phenomena. For example, Liu et al. recently demonstrated a universal mapping of topological singularities from momentum to real space [64]. Yang et al. demonstrated a 3D photonic topological insulator with an extremely wide topological bandgap [65]. In particular, non-Abelian gauge fields in real space, non-Abelian nodal links in momentum space, braiding topological zero modes have also been observed in photonic experiments very recently [66-68]. Some recent progresses in this area are reported in a special collection of Light Science \& Applications [69].

In parallel, another burgeoning area in the last decade is the parity-time $(P T)$ symmetry optics, or more generally, non-Hermitian photonics. In 2008, the concept of $P T$-symmetry developed in quantum mechanics was first integrated into the domain of optics and photonics by Makris et al. [70], and independently by Kleiman et al. [71] who introduced the concept of exceptional points into photonics. Soon thereafter, $P T$-symmetry and exceptional points were demonstrated in both passive [72] and active [73] photonic systems, by manipulation of optical gain and loss (analogous to complex quantum potentials), from two-component $P T$ couplers $[72,73]$ to synthetic $P T$ lattices [74]. Subsequently, many surprising experiments and fundamental findings blossomed in the study of non-Hermitian photonic systems, including for example $P T$-symmetric microring lasers and whisperinggallery microcavities [75-77], and the use of the extreme sensitivity near exceptional points for sensing [78-80]. Ever since these important experimental demonstrations, non-Hermitian physics has turned into one of the fast growing and important research frontiers in the field of photonics and beyond [81-84].

Perhaps, one of the most important developments in the broader field of topological/non-Hermitian photonics came with the proposal of topological insulator lasers [85], which was made more than a year before any suggestion or experiment to combine the concepts of topology and lasing. Undoubtedly, in the entire field of topological photonics, the research topic that is closest to real technological application is the topological insulator lasers. The motivation was to harness the features of topologically protected transport to force many semiconductor emitters to lock together and behave as a single powerful highly coherent laser source [85]. Technologically, having a high-power semiconductor laser has been a challenge of more than four decades, and all attempts to make a coherent "broad-area laser" or a "laser diode array" have generally failed. Laser arrays are currently used only as a strong flashlight to pump solid state lasers (NdYag etc.), but their coherence is not much better than of a light emitting diode. The vision-from the first time this idea was proposed [85] — was therefore to make use of the fundamental features of topological insulators to force injection-locking of many semiconductor laser emitters to act as a single coherent laser. However, because a laser system is inherently a non-Hermitian system which includes gain and loss, there stood out a question whether or not a non-Hermitian system can support any kind of topological protection.

Combining topology with non-Hermitian physics seems to be natural but conceptually it is a highly challenging goal. The first non-Hermitian topological system was demonstrated in a photonics experiment [86], following an earlier prediction of a topological transition in non-Hermitian quantum walk [87]. This was a onedimensional (1D) non-Hermitian lattice where the presence of loss made it possible to identify the topology of the corresponding passive non-Hermitian system just from bulk measurements, without the need to investigate edge states. Notwithstanding that experiment, the idea of combining topology and non-Hermitian physics was bound to create controversies and arguments: at the very least, topological physics relies on the existence of topological quantities that remain invariant during deformations of the system, yet in non-Hermitian systems it was not clear at all that such conserved quantities can exist. In fact, there were some theoretical papers that claimed, for example, that $P T$-symmetric topological systems cannot exist, casting major doubts on the ability of nonHermitian systems to exhibit topologically-protected transport. Some of the controversial issues were resolved by the demonstration of $1 \mathrm{D}$ topological photonic systems exhibiting broken [88] and full [89] PT-symmetry. However, these 1D systems cannot have topologicallyprotected transport along their edge, because the edge in a $1 \mathrm{D}$ system is zero-dimensional. As such, these experiments $[88,89]$ and the theory therein do not really provide an unequivocal proof for the existence of conserved topological properties in non-Hermitian systems. Taking the controversial idea a step forward and suggesting the topological insulator lasers has created more controversies: namely, lasers generally combine gain, cavity and the nonlinearity of gain saturation. The proposal for the topological insulator laser [85] relied on two important topological features: robustness against random variations in the on-site potential, and the non-zero group velocity of the topological edge modes. The former property is essential to ensure injection-locking of multiple laser elements that could afford deviations in their resonance 
frequencies (inevitable in the fabrication process); and the latter guarantees that the lasing topological edge mode includes all the elements and does not allow independent lasing of local groups of emitters. Nonetheless, despite the early vision in 2016 [85], the idea initially met with much skepticism. Even when a topological insulator laser was first experimentally demonstrated [90], and even now-4 years after-the issue of topological protection of transport in a non-Hermitian system is not fully resolved. Exactly because of this issue, the first theoretical model proposed for topological insulator lasers was chosen to be based on the archetypical Haldane model with the addition of gain, loss and nonlinearity [91]. The simulations showed unequivocally that the topological platform gives rise to immune transport in this $2 \mathrm{D}$ laser system despite the non-Hermiticity. This led to the conjecture that topological invariants should exist also in non-Hermitian systems, as otherwise there would be no explanation to the unidirectional transport and the immunity to defects and disorder [90]. This was the goal of several recent papers [92-96] to present a general framework for classifying topological phases of generic non-Hermitian systems. This fundamental issue is still one of the outstanding challenges in topological physics at large, where topological photonics is the spearhead of research.

Back to the topological insulator lasers (leaving behind the questions about combining topology and non-Hermitian physics), after the first suggestion of topological lasers [85], there was a series of early works demonstrating laser emitting from a zero-dimensional topological edge state in a 1D chain [97-99]. But in those systems there was no edge transport at all, because the lasing was almost fully confined to a single resonator. Then, there was an attempt to incorporate gyro-optic material in a laser cavity [100], but since the magneto-optic effects at optical frequencies are extremely weak, the bandwidth of the laser was broader than the topological bandgap and there was no evidence of protected transport. Eventually, the first topological insulator laser was demonstrated theoretically [91] and experimentally [101] (Fig. 2a), displaying the expected features. This laser was constructed on a standard optoelectronic platform, as an aperiodic array of $10 \times 10$ coupled ring-resonators on InGaAsP quantum wells wafer. This $2 \mathrm{D}$ setting is composed of a square lattice of ring resonators coupled to each other via auxiliary links. The intermediary links are judiciously spatially shifted to introduce a set of hopping phases, establishing a synthetic magnetic field that yields topological features. To promote lasing of the topologicallyprotected edge modes, only the outer perimeter of the array was pumped, while leaving the interior elements lossy. This topological insulator laser operated in single mode, even considerably above threshold, whereas the corresponding topologically trivial realizations led to lasing in an undesired multimode fashion. More importantly, the topological laser displayed a slope efficiency that was considerably higher than in the corresponding trivial realizations, even in the presence of defects and disorder.

Since then, several groups followed with a variety of configurations for realizing topological insulator lasers, e.g., a topological quantum cascade laser with valley edge modes [102] (Fig. 2b), a topological bulk laser based on band-inversion-induced reflection [103], a topological nanolasers based on second-order corner states [104], a topological insulator laser with next-nearest-neighbor coupling [105], and a Dirac-vortex topological cavity promising for large-area single-mode lasing [106]. Finally, we note the very recent experiments on a topological
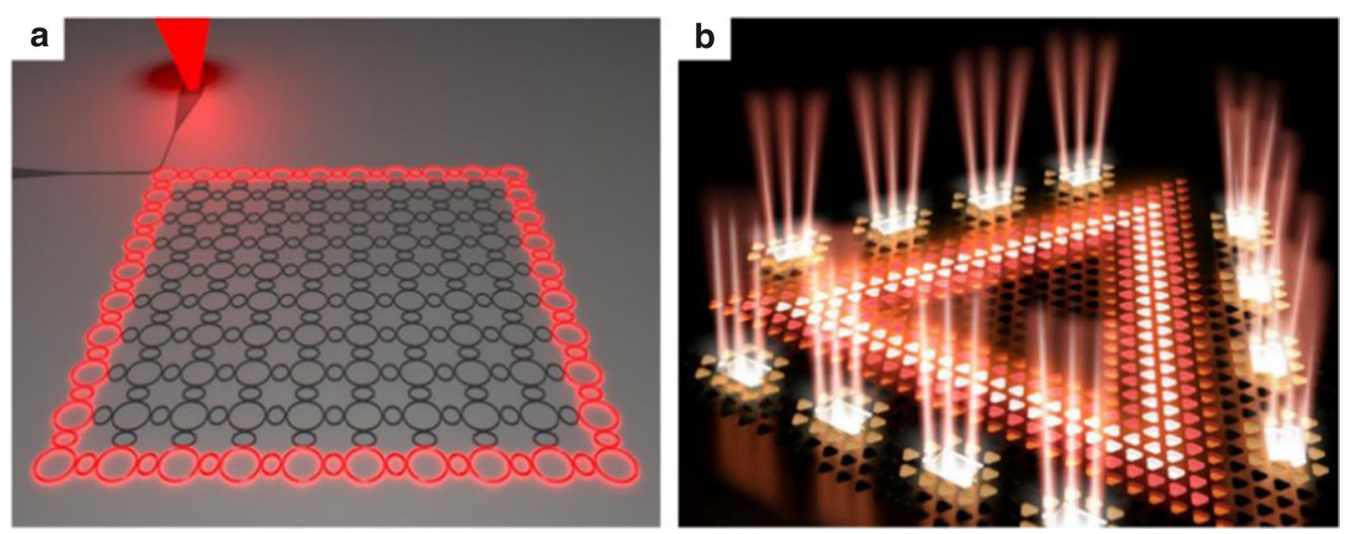

Fig. 2 a Artistic illustration of the first demonstrated topological insulator laser, where lasing goes around the perimeter unobstructed by sharp corners or disorder, exiting from the output port [91, 101] (Credit: Miguel Bandres, UCF). b Artist's impression of an electrically-driven topological quantum cascade laser, established based on the valley-Hall edge modes [102] (Credit: Songdi Tech Co, Ltd) 
vertical cavity surface emitting laser [107]. On the theoretical side, recent analysis [108] on the coherence of a topological insulator laser has shown that indeed the topological landscape greatly improves the mutual coherence even with a large number of emitters. Thus, it seems that the goal of locking together many semiconductor lasers to act as a single coherent laser will be achieved and is well on its way to become a real application. From all of this activity, it is now quite clear that currently the topological insulator lasers are the most promising application of topological photonics, with many new ideas emerging, for example, utilizing topology in synthetic dimensions to force an array of semiconductor lasers to emit mode-locked pulses [109], which could overcome a challenge of three decades.

\section{Al-empowered nanophotonics and photonic machine-learning}

In the past decades, we have witnessed a mind-blowing rise of artificial intelligence (AI)-based technologies that are impacting numerous industrious areas from autonomous driving, health care, to advanced manufacturing and energy harvesting. The advancement of AI, especially its subfield of machine learning, has revolutionized many fields of science and engineering including speech and visual object recognition and computer vision. In physical sciences, for example, the concepts and methods of machine learning have been developed and applied in a variety of branches such as particle physics and cosmology, quantum physics and quantum computing, computational chemistry and materials design [110]. Nevertheless, it is perhaps our increased everyday experience with the success of machine learning techniques in cameras, smartphones, video games, robots and selfdriving cars that really influenced our perception on what AI can and might do for us.

In parallel, nanophotonics as a rising field devoted to the study of nanoscale light-matter interaction has advanced tremendously, broadly defined and inherently integrated into different photonic realms including photonic crystals, plasmonics, metamaterials and metasurfaces, metaphotonics, 2D material photonics, apart from quantum photonics and topological photonics mentioned earlier. Due to rapid progress of nanoscale fabrication techniques, different synthetic photonic materials emerge constantly that exhibit extraordinary properties not found from naturally occurring materials, providing ultimate opportunities for shaping and manipulation of light. Advances in nanophotonics have certainly led to new fundamental understandings as well as a wide variety of important applications, as have already been demonstrated for flat optics, integrated silicon photonics, transparent display, nanophotonic color technologies, thermal radiation control, plasmonic nanolasers, to name just several [111-124].

As the design for photonic materials and nanostructures becomes more and more sophisticated, challenging and overwhelming, new computational methodology and techniques are steadily sought after by researchers. The blending between machine learning techniques and nanophotonics came naturally, and has thus attracted a great deal of ever-increasing interest in various aspects of light science and applications. The design and simulation of photonic systems often involve computational electromagnetics that can greatly benefit from the core strength of neural networks. As such, deep learning, a subset of machine learning that relies on multi-layers of neural networks learned from data rather than designed by human experts [125], is making rapid advances in solving sophisticated photonics tasks. Due to the fundamental change of deep learning algorithms along with their tremendous potential, such a data-driven technique has led to an explosion of efforts for inverse design of new photonic materials as well as for exploitation of novel nanophotonic devices, as discussed in recent reviews [126-129].

Indeed, although there was a great deal of interest in optical artificial neural networks [130] and optical computing [131] some decades ago, the prospect of their practical implementations has been reinvigorated only recently, thanks to rapid development of the machine learning techniques and the nanophotonic technologies. AI-empowered photonics is drawing increasing attention from a growing community of researchers worldwide, with surprising discoveries reported frequently. For example, Shen \& Harris et al. explored a fully-optical architecture for implementing general deep learning algorithms using silicon-based nanophotonic circuits, aiming for light-powered computers [132] (Fig. 3a). Hughes et al. found a method to train artificial neural networks directly on a photonic chip [133], targeting to optical circuits that can perform neural network computations more efficiently than electronic digital computers. Lin et al. introduced an all-optical deep learning framework in which the artificial neural networks is physically formed by multiple layers of diffractive optical elements that could be trained to execute complex functions at the speed of light [134], where the passive optical components used for such all-optical machine learning were patterned and fabricated with 3D-printing, and the device could be readily scaled up using various high-throughput and large-area 3D-fabrication methods. Feldmann et al. presented an all-optical version of spiking neurosynaptic networks with selflearning capabilities [135], and exploited the wavelengthdivision-multiplexing techniques to implement a scalable 

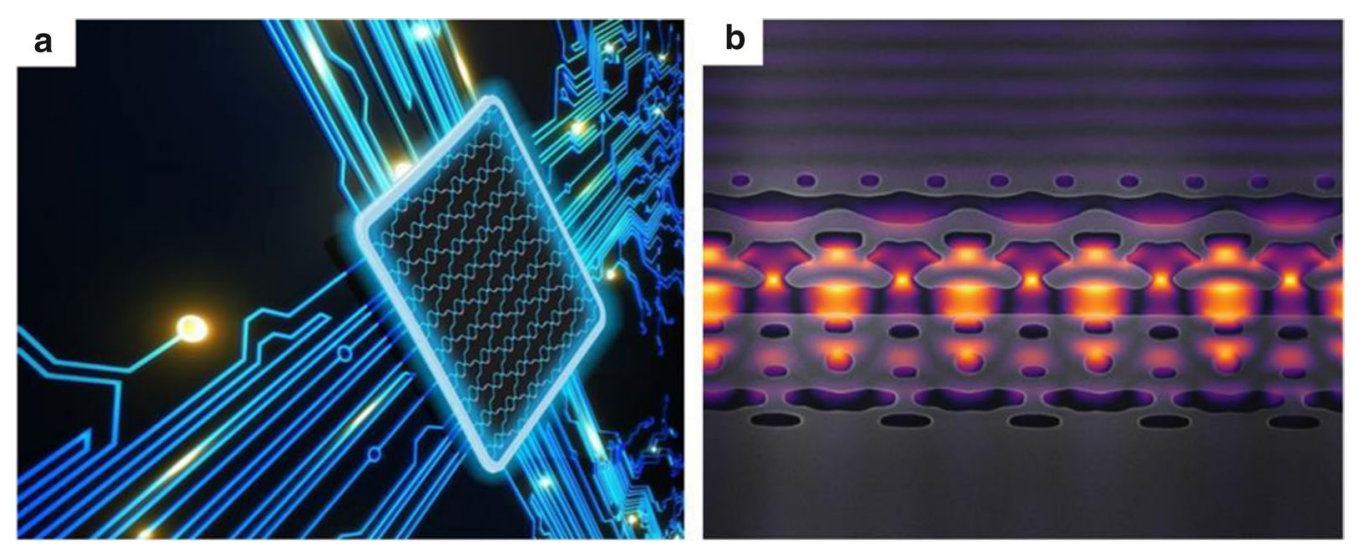

Fig. 3 a A picture of programmable nanophotonic processors integrated on a printed circuit board for carrying out deep learning computing [132] (Credit: MIT); b A magnified image showing an inverse-design-assisted on-chip integrated laser-driven particle accelerator. The oddly shaped gray structures are nanometer-sized features carved in to silicon that focus bursts of infrared laser light, shown in yellow and purple, on a flow of electrons through the center channel. By packing many such acceleration channels onto an inch-sized chip, an electron beam is expected to be accelerated close to the speed of light [145] (Credit: Neil Sapra)

circuit architecture for photonic neural networks and demonstrated pattern recognition directly in the optical domain. Such all-optical neurons are expected to ideally mimic the biological neural networks but could in principle operate several orders of magnitude faster. In yet another recent endeavor, Mennel et al. demonstrated an artificial-neural-network-based vision sensor for ultrafast recognition and encoding of optical images projected onto the chip of a reconfigurable $2 \mathrm{D}$ semiconductor photodiode array [136]. Such a device can be easily scalable, promising for various training possibilities for ultrafast machine vision applications.

These and many other advances in deep learning photonics may herald the advent of practical photonic chips that could outshine the conventional chips with faster and more energy efficient ways to perform complex tasks. Needless to say, the AI-empowered advancements have accelerated dramatically in a broad spectrum of nanophotonics and related fields, from design of novel metasurfaces to emulation of biological neural networks, and from materials discovery, optical characterizations to photonics technologies mediated by light-matter interaction [137-142]. It is truly amazing to see how the inverse design technique for arbitrary nanophotonic structures [143] has led to truly exciting applications such as chip-based LiDAR [144] and on-chip laser-driven particle accelerators [145] (Fig. 3b). Many equally exciting applications with deep learning in other areas are continuing to emerge, from intelligent metadevices for self-adaptive cloak [146] to microscopy and cellular image analysis $[147,148]$. As the level of activity and the enthusiasm centered around this interdisciplinary field continue to grow, we envision more exhilarating results and resounding successes will stem from the interplay between photonics-enabled neural networks and AIempowered nanophotonics.

\section{Looking into the future}

In the sections above, we have only provided a glimpse of just three photonic subareas that have grown tremendously in the past decade, and that we believe will continue to advance in coming years. While each of these three areas has its own feature and focus, they are strongly connected and intertwined. In fact, research in quantum photonics, topological photonics, and machine learning photonics all crosses multiple disciplines, united by the ultimate goal to better control and use light for practical applications. For examples, quantum photonic devices and quantum computing can benefit greatly from nanophotonic technologies and machine learning techniques, as already mentioned above. The concept of topological photonics, on the other hand, has also been developed and applied into the other areas, leading to subdisciplines such as topological quantum photonics that aims for topological protection of photonic quantum states and their transport [149-153], and light interaction with topological nanostructures and robust control of light in topological photonic nanostructures [154158]. Meanwhile, machine learning techniques have recently been applied to solve problems in topological photonics and phononics [159-161], and to reconstruct quantum states of photons from experimental data [162], apart from many applications in programmable photonic circuits, optical computing and facial image recognition [163-165]. Undoubtedly, as new machine learning algorithms and architectures are developed to surpass 
human performance in a variety of tasks, their applications in quantum photonics and topological nanophotonics will continue to blossom, which could lead to flexible and unconventional control of light. We envisage much is to be accomplished in these closely coupled and dynamically developing photonic areas.

The major progresses in these and other photonics areas had and will continue to have a profound impact on fundamental physics at large. Some of these have already mentioned in the sections above. For example, the entire progress on non-Hermitian topological physics, with its still challenging questions, has been pioneered in photonics and led by photonics. Likewise, in the topologically-protected transport of quantum states, the successful experiments accomplished thus far are in photonics. In a similar vein, experiments on topological Anderson insulators [166] have been pioneered also in photonics, and only later on followed by experiments in condensed matter. It is already clear that photonics plays a major role now in exploring new fundamental concepts, as well as revisiting some of the mind-boggling peculiarities of the quantum world.

Looking into the future, there are also many challenges in these photonic areas. For instance, as mentioned at the beginning, realization and development of integrated quantum photonic technologies are crucial for scalable quantum applications such as quantum computing and information processing. To achieve such applications, major challenges include for example realization of a reliable source of cluster states, on-chip integration of efficient single-photon sources, and development of large-scale nanoassembly of quantum sources and quantum circuitries. Finding the solutions to address some of these challenges could be assisted by applying advanced machine learning techniques to conventional methods in quantum photonics, as highlighted in a recent perspective article [167]. In the area of topological/non-Hermitian photonics, on the other hand, there are also challenges in both fundamentals and applications. For examples, is there a general theory to characterize a complex system with underlying physics driven simultaneously by nonlinearity, topology and non-Hermiticity, and how would nonlinearity affect overall topological features and the global classifications of symmetry and topology $[91,168,169]$ ? The topological insulator lasers seem to be the most promising application of topological photonics, but it is yet unknown if the idea of topologically locking laser arrays would work at a large scale, e.g., for 1000 emitters or more. Should some of these topological applications become real technology, that means they will also commercially outperform the available photonic crystal surface-emitting lasers (PCSELs) and semiconductor vertical cavity surface-emitting lasers
(VCSELs), it could overcome the challenges of many decades $[63,106,107]$.

\section{Conclusions}

In lieu of a conclusion, we just want to point out that there are many other areas in photonics that we believe will continue to rise in coming years, such as photonic quantum simulators, photovoltaics, flexible photonic display, metasurface-empowered flat photonics, VCSELs, intelligent biophotonics and ultrafast nanoscopy. Further advances in fundamental research and technological innovation in photonics could unleash the extraordinary potential of the science of light and its impact to our society in the next generation. In particular, the future impact arising from incredible advancements in artificial intelligence and related photonic technologies could be overwhelming. We envision that the science of light in general will continue to transform some major areas where photonics-based solutions would play the key roles, actively or serendipitously, lighting and shaping our pathway towards a brighter future.

\section{Acknowledgements}

We wish to thank Netanel Lindner of the Technion for his illuminating comments on the section on photonic quantum computing.

\section{Authors' contributions}

Both authors read and approved the final manuscript.

\section{Funding}

ZC acknowledges the support from the National Key R\&D Program of China under Grant (No. 2017YFA0303800). MS acknowledges support from the Israel Science Foundation.

Availability of data and materials

Not applicable.

\section{Declarations}

Competing interests

There is no competing interests for this article.

\section{Author details}

${ }^{1}$ The MOE Key Laboratory of Weak-Light Nonlinear Photonics, TEDA Applied Physics Institute and School of Physics, Nankai University, Tianjin 300457, China. ${ }^{2}$ Department of Physics and Astronomy, San Francisco State University, San Francisco, CA 94132, USA. ${ }^{3}$ Physics Department, Electrical Engineering

Department, and Solid State Institute, Technion, Haifa, Israel.

Received: 19 December 2020 Revised: 13 March 2021 Accepted: 24 March 2021

Published online: 08 June 2021

\footnotetext{
References

1. A. Aspect, P. Grangier, G. Roger, Experimental realization of EinsteinPodolsky-Rosen-Bohm gedankenexperiment: a new violation of Bell's inequalities. Phys. Rev. Lett. 49, 91-94 (1982)

2. S.J. Freedman, J.F. Clauser, Experimental test of local hidden-variable theories. Phys. Rev. Lett. 28, 938-941 (1972)

3. M.H. Anderson, J.R. Ensher, M.R. Matthews, C.E. Wieman, E.A. Cornell, Observation of Bose-Einstein condensation in a dilute atomic vapor Science 269, 198-201 (1995)
} 
4. M. Greiner, O. Mandel, T. Esslinger, T.W. Hansch, I. Bloch, Quantum phase transition from a superfluid to a Mott insulator in a gas of ultracold atoms. Nature 415, 39-44 (2002)

5. A. McPherson et al., Studies of multiphoton production of vacuumultraviolet radiation in the rare gases. J. Opt. Soc. Am. B 4, 595-601 (1987)

6. P.B. Corkum, Plasma perspective on strong field multiphoton ionization. Phys. Rev. Lett. 71, 1994-1997 (1993)

7. M. Hentschel et al., Attosecond metrology. Nature 414, 509-513 (2001)

8. S.W. Hell, J. Wichmann, Breaking the diffraction resolution limit by stimulated emission: stimulated-emission-depletion fluorescence microscopy. Opt. Lett. 19, 780-782 (1994)

9. J.L. O'Brien, A. Furusawa, J. Vučković, Photonic quantum technologies Nat. Photon. 3, 687-695 (2009)

10. D.M. Lukin, M.A. Guidry, J. Vučković, Integrated quantum photonics with silicon carbide: challenges and prospects. PRX Quantum 1, 020102 (2020)

11. F. Xu, X. Ma, Q. Zhang, H.-K. Lo, J.-W. Pan, Secure quantum key distribution with realistic devices. Rev. Mod. Phys. 92, 025002 (2020)

12. J. Yin et al., Entanglement-based secure quantum cryptography over 1,120 kilometres. Nature 582, 501-505 (2020)

13. L. Greenemeier. How close are we-really-to building a quantum computer? in Scientific American (May 30, 2018)

14. J. Wang, F. Sciarrino, A. Laing, M.G. Thompson, Integrated photonic quantum technologies. Nat. Photon. 14, 273-284 (2020)

15. A.W. Elshaari, W. Pernice, K. Srinivasan, O. Benson, V. Zwiller, Hybrid integrated quantum photonic circuits. Nat. Photon. 14, 285-298 (2020)

16. F. Peyskens, C. Chakraborty, M. Muneeb, D. Van Thourhout, D. Englund, Integration of single photon emitters in $2 \mathrm{D}$ layered materials with a silicon nitride photonic chip. Nat. Commun. 10, 4435 (2019)

17. S. Paesani et al., Near-ideal spontaneous photon sources in silicon quantum photonics. Nat. Commun. 11, 2505 (2020)

18. D.M. Lukin et al., $4 \mathrm{H}$-silicon-carbide-on-insulator for integrated quantum and nonlinear photonics. Nat. Photon. 14, 330-334 (2020)

19. E.M.T. Fadaly et al., Direct-bandgap emission from hexagonal Ge and SiGe alloys. Nature 580, 205-209 (2020)

20. N. Akopian et al., Entangled photon pairs from semiconductor quantum dots. Phys. Rev. Lett. 96, 130501 (2006)

21. I. Schwartz et al., Deterministic generation of a cluster state of entangled photons. Science 354, 434-437 (2016)

22. P. Lodahl, S. Mahmoodian, S. Stobbe, Interfacing single photons and single quantum dots with photonic nanostructures. Rev. Mod. Phys. 87, 347-400 (2015)

23. T. Peyronel et al., Quantum nonlinear optics with single photons enabled by strongly interacting atoms. Nature 488, 57-60 (2012)

24. D.E. Chang, V. Vuletić, M.D. Lukin, Quantum nonlinear optics - photon by photon. Nat. Photon. 8, 685-694 (2014)

25. J. Benhelm, G. Kirchmair, C.F. Roos, R. Blatt, Towards fault-tolerant quantum computing with trapped ions. Nat. Phys. 4, 463-466 (2008)

26. S. Debnath et al., Demonstration of a small programmable quantum computer with atomic qubits. Nature 536, 63-66 (2016)

27. R.J. Niffenegger et al., Integrated multi-wavelength control of an ion qubit. Nature 586, 538-542 (2020)

28. R. Raussendorf, H.J. Briegel, A one-way quantum computer. Phys. Rev. Lett. 86, 5188-5191 (2001)

29. T. Rudolph, Why I am optimistic about the silicon-photonic route to quantum computing. APL Photon. 2, 030901 (2017)

30. H.S. Zhong et al., Quantum computational advantage using photons. Science $\mathbf{3 7 0}$, eabe8770 (2020)

31. H. Bernien et al., Probing many-body dynamics on a 51 -atom quantum simulator. Nature 551, 579-584 (2017)

32. A. Sipahigil et al., An integrated diamond nanophotonics platform for quantum-optical networks. Science 354, 847-850 (2016)

33. P. Walther et al., Experimental one-way quantum computing. Nature 434, 169-176 (2005)

34. C. Reimer et al., High-dimensional one-way quantum processing implemented on d-level cluster states. Nat. Phys. 15, 148-153 (2019)

35. W. Asavanant et al., Generation of time-domain-multiplexed twodimensional cluster state. Science 366, 373-376 (2019)
36. M.V. Larsen, X. Guo, C.R. Breum, J.S. Neergaard-Nielsen, U.L. Andersen, Deterministic generation of a two-dimensional cluster state. Science 366, 369-372 (2019)

37. F.D. Haldane, S. Raghu, Possible realization of directional optical waveguides in photonic crystals with broken time-reversal symmetry. Phys. Rev. Lett. 100, 013904 (2008)

38. Z. Wang, Y. Chong, J.D. Joannopoulos, M. Soljacic, Observation of unidirectional backscattering-immune topological electromagnetic states. Nature 461, 772-775 (2009)

39. M.C. Rechtsman et al., Photonic Floquet topological insulators. Nature 496, 196-200 (2013)

40. N.H. Lindner, G. Refael, V. Galitski, Floquet topological insulator in semiconductor quantum wells. Nat. Phys. 7, 490-495 (2011)

41. M. Hafezi, S. Mittal, J. Fan, A. Migdall, J.M. Taylor, Imaging topological edge states in silicon photonics. Nat. Photon. 7, 1001-1005 (2013)

42. A.B. Khanikaev et al., Photonic topological insulators. Nat. Mater. 12 233-239 (2013)

43. W.J. Chen et al., Experimental realization of photonic topological insulator in a uniaxial metacrystal waveguide. Nat. Commun. 5, 5782 (2014)

44. T. Ma, G. Shvets, All-Si valley-Hall photonic topological insulator. New J. Phys. 18, 025012 (2016)

45. L.H. Wu, X. Hu, Scheme for achieving a topological photonic crystal by using dielectric material. Phys. Rev. Lett. 114, 223901 (2015)

46. G.Q. Liang, Y.D. Chong, Optical resonator analog of a two-dimensional topological insulator. Phys. Rev. Lett. 110, 203904 (2013)

47. F. Gao et al., Probing topological protection using a designer surface plasmon structure. Nat. Commun. 7, 11619 (2016)

48. L. Yuan, Y. Shi, S. Fan, Photonic gauge potential in a system with a synthetic frequency dimension. Opt. Lett. 41, 741-744 (2016)

49. T. Ozawa, H.M. Price, N. Goldman, O. Zilberberg, I. Carusotto, Synthetic dimensions in integrated photonics: from optical isolation to fourdimensional quantum Hall physics. Phys. Rev. A 93, 043827 (2016)

50. E. Lustig et al., Photonic topological insulator in synthetic dimensions. Nature 567, 356-360 (2019)

51. M. Mancini et al., Observation of chiral edge states with neutral fermions in synthetic Hall ribbons. Science 349, 1510-1513 (2015)

52. B.K. Stuhl, H.I. Lu, L.M. Aycock, D. Genkina, I.B. Spielman, Visualizing edge states with an atomic Bose gas in the quantum Hall regime. Science 349, 1514-1518 (2015)

53. D. Jukić, H. Buljan, Four-dimensional photonic lattices and discrete tesseract solitons. Phys. Rev. A 87, 013814 (2013)

54. X.W. Luo et al., Quantum simulation of 2D topological physics in a 1D array of optical cavities. Nat. Commun. 6, 7704 (2015)

55. H.M. Price, O. Zilberberg, T. Ozawa, I. Carusotto, N. Goldman, Fourdimensional quantum hall effect with ultracold atoms. Phys. Rev. Lett. 115, 195303 (2015)

56. M.A. Bandres, M.C. Rechtsman, M. Segev, Topological photonic quasicrystals: fractal topological spectrum and protected transport. Phys. Rev. X 6, 011016 (2016)

57. Z. Yang, E. Lustig, Y. Lumer, M. Segev, Photonic Floquet topological insulators in a fractal lattice. Light. Sci. Appl. 9, 128 (2020)

58. S. Klembt et al., Exciton-polariton topological insulator. Nature 562, 552-556 (2018)

59. L. Lu, J.D. Joannopoulos, M. Soljačić, Topological photonics. Nat. Photon. 8. $821-829(2014)$

60. A.B. Khanikaev, G. Shvets, Two-dimensional topological photonics. Nat. Photon. 11, 763-773 (2017)

61. T. Ozawa et al., Topological photonics. Rev. Mod. Phys. 91, 015006 (2019)

62. D. Smirnova, D. Leykam, Y. Chong, Y. Kivshar, Nonlinear topological photonics. Appl. Phys. Rev. 7, 021306 (2020)

63. M. Segev, M.A. Bandres, Topological photonics: where do we go from here? Nanophotonics 10, 425-434 (2021)

64. X. Liu et al., Universal momentum-to-real-space mapping of topological singularities. Nat. Commun. 11, 1568 (2020)

65. Y. Yang et al., Realization of a three-dimensional photonic topological insulator. Nature 565, 622-626 (2019)

66. Y. Yang et al., Synthesis and observation of non-Abelian gauge fields in real space. Science 365, 1021-1025 (2019)

67. E. Yang et al., Observation of non-Abelian nodal links in photonics. Phys. Rev. Lett. 125, 033901 (2020) 
68. J. Noh et al., Braiding photonic topological zero modes. Nat. Phys. 16, 989-993 (2020)

69. Z. Chen, D. Leykam, H. Buljan. (eds) Special Issue on Topological photonics and beyond: novel concepts and recent advances. Light. Sci. Appl. 9, 203 (2020).

70. K.G. Makris, R. El-Ganainy, D.N. Christodoulides, Z.H. Musslimani, Beam dynamics in PT symmetric optical lattices. Phys. Rev. Lett. 100, 103904 (2008)

71. S. Klaiman, U. Gunther, N. Moiseyev, Visualization of branch points in PT-symmetric waveguides. Phys. Rev. Lett. 101, 080402 (2008)

72. A. Guo et al., Observation of PT-symmetry breaking in complex optical potentials. Phys. Rev. Lett. 103, 093902 (2009)

73. C.E. Rüter et al., Observation of parity-time symmetry in optics. Nat. Phys. 6, 192-195 (2010)

74. A. Regensburger et al., Parity-time synthetic photonic lattices. Nature 488, 167-171 (2012)

75. H. Hodaei, M.A. Miri, M. Heinrich, D.N. Christodoulides, M. Khajavikhan, Parity-time-symmetric microring lasers. Science 346, 975-978 (2014)

76. L. Feng, Z.J. Wong, R.M. Ma, Y. Wang, X. Zhang, Single-mode laser by parity-time symmetry breaking. Science 346, 972-975 (2014)

77. B. Peng et al., Parity-time-symmetric whispering-gallery microcavities. Nat. Phys. 10, 394-398 (2014)

78. H. Hodaei et al., Enhanced sensitivity at higher-order exceptional points. Nature 548, 187-191 (2017)

79. W. Chen, S. Kaya Ozdemir, G. Zhao, J. Wiersig, L. Yang, Exceptional points enhance sensing in an optical microcavity. Nature 548, 192-196 (2017)

80. M.P. Hokmabadi, A. Schumer, D.N. Christodoulides, M. Khajavikhan, Non-Hermitian ring laser gyroscopes with enhanced Sagnac sensitivity. Nature 576, 70-74 (2019)

81. L. Feng, R. El-Ganainy, L. Ge, Non-Hermitian photonics based on paritytime symmetry. Nat. Photon. 11, 752-762 (2017)

82. R. El-Ganainy et al., Non-Hermitian physics and PT symmetry. Nat. Phys. 14, 11-19 (2018)

83. S.K. Ozdemir, S. Rotter, F. Nori, L. Yang, Parity-time symmetry and exceptional points in photonics. Nat. Mater. 18, 783-798 (2019)

84. M. Parto, Y.G.N. Liu, B. Bahari, M. Khajavikhan, D.N. Christodoulides, NonHermitian and topological photonics: Optics at an exceptional point. Nanophotonics 10, 403-423 (2020)

85. G. Harari et al. Topological lasers. in Conference on Lasers and ElectroOptics (OSA Technical Digest paper FM3A.3, San Jose, California, United States, 2016).

86. J.M. Zeuner et al., Observation of a topological transition in the bulk of a non-Hermitian system. Phys. Rev. Lett. 115, 040402 (2015)

87. M.S. Rudner, L.S. Levitov, Topological transition in a non-Hermitian quantum walk. Phys. Rev. Lett. 102, 065703 (2009)

88. C. Poli, M. Bellec, U. Kuhl, F. Mortessagne, H. Schomerus, Selective enhancement of topologically induced interface states in a dielectric resonator chain. Nat. Commun. 6, 6710 (2015)

89. S. Weimann et al., Topologically protected bound states in photonic parity-time-symmetric crystals. Nat. Mater. 16, 433-438 (2017)

90. S. Wittek et al. Towards the experimental realization of the topological insulator laser. in Conference on Lasers and Electro-Optics (OSA Technical Digest paper FTh1D.3, San Jose, California, United States, 2017).

91. G. Harari et al., Topological insulator laser: Theory. Science 359, eaar4003 (2018)

92. Z. Gong et al., Topological phases of non-Hermitian systems. Phys. Rev. $X \mathbf{8}, 031079(2018)$

93. H. Shen, B. Zhen, L. Fu, Topological band theory for non-Hermitian Hamiltonians. Phys. Rev. Lett. 120, 146402 (2018)

94. L. Xiao et al., Non-Hermitian bulk-boundary correspondence in quantum dynamics. Nat. Phys. 16, 761-766 (2020)

95. K. Zhang, Z. Yang, C. Fang, Correspondence between winding numbers and skin modes in non-Hermitian systems. Phys. Rev. Lett. 125, 126402 (2020)

96. T. Helbig et al., Generalized bulk-boundary correspondence in nonHermitian topolectrical circuits. Nat. Phys. 16, 747-750 (2020)

97. P. St-Jean et al., Lasing in topological edge states of a one-dimensional lattice. Nat. Photon. 11, 651-656 (2017)

98. H. Zhao et al., Topological hybrid silicon microlasers. Nat. Commun. 9, 981 (2018)
99. M. Parto et al., Edge-mode lasing in $1 \mathrm{D}$ topological active arrays. Phys. Rev. Lett. 120, 113901 (2018)

100. B. Bahari et al., Nonreciprocal lasing in topological cavities of arbitrary geometries. Science 358, 636-640 (2017)

101. M.A. Bandres et al., Topological insulator laser: Experiments. Science 359, eaar4005 (2018)

102. Y. Zeng et al., Electrically pumped topological laser with valley edge modes. Nature 578, 246-250 (2020)

103. Z.K. Shao et al., A high-performance topological bulk laser based on band-inversion-induced reflection. Nat. Nanotechnol. 15, 67-72 (2020)

104. W. Zhang et al., Low-threshold topological nanolasers based on the second-order corner state. Light Sci. Appl. 9, 109 (2020)

105. Y.G. Liu et al. Towards the experimental demonstration of topological Haldane lattice in microring laser arrays (Conference Presentation). in Proc. SPIE. 11301, Novel In-Plane Semiconductor Lasers XIX (SPIE, San Francisco, California, United States, 2020).

106. X. Gao et al., Dirac-vortex topological cavities. Nat. Nanotechnol. 15, 1012-1018 (2020)

107. A. Dikopoltsev et al. Topological insulator VCSEL array. in Conference on Lasers and Electro-Optics (OSA Technical Digest paper FM1A.4, Washington, DC United States, 2020).

108. I. Amelio, I. Carusotto. Theory of the coherence of topological lasers. Preprint at https://arxiv.org/abs/1911.10437 (2020).

109. Z. Yang et al., Mode-locked topological insulator laser utilizing synthetic dimensions. Phys. Rev. X 10, 011059 (2020)

110. G. Carleo et al., Machine learning and the physical sciences. Rev. Mod. Phys. 91, 045002 (2019)

111. R. Won, Integrating silicon photonics. Nat. Photon. 4, 498-499 (2010)

112. D.J. Moss, R. Morandotti, A.L. Gaeta, M. Lipson, New CMOS-compatible platforms based on silicon nitride and Hydex for nonlinear optics. Nat. Photon. 7, 597-607 (2013)

113. A.V. Kildishev, A. Boltasseva, V.M. Shalaev, Planar photonics with metasurfaces. Science 339, 1232009 (2013)

114. N. Yu, F. Capasso, Flat optics with designer metasurfaces. Nat. Mater. 13, 139-150 (2014)

115. C.W. Hsu et al., Transparent displays enabled by resonant nanoparticle scattering. Nat. Commun. 5, 3152 (2014)

116. A.F. Koenderink, A. Alu, A. Polman, Nanophotonics: shrinking lightbased technology. Science 348, 516-521 (2015)

117. D. Thomson et al., Roadmap on silicon photonics. J. Opt. 18, 073003 (2016)

118. A.I. Kuznetsov, A.E. Miroshnichenko, M.L. Brongersma, Y.S. Kivshar, B. Luk'yanchuk, Optically resonant dielectric nanostructures. Science $\mathbf{3 5 4}$ aag2472 (2016)

119. A. Kristensen et al., Plasmonic colour generation. Nat. Rev. Mater $\mathbf{2}$, 16088 (2016)

120. P. Cheben, R. Halir, J.H. Schmid, H.A. Atwater, D.R. Smith, Subwavelength integrated photonics. Nature 560, 565-572 (2018)

121. A.P. Raman, M.A. Anoma, L. Zhu, E. Rephaeli, S. Fan, Passive radiative cooling below ambient air temperature under direct sunlight. Nature 515, 540-544 (2014)

122. O. Ilic et al., Tailoring high-temperature radiation and the resurrection of the incandescent source. Nat. Nanotechnol. 11, 320-324 (2016)

123. Z. Dai et al., Artificial metaphotonics born naturally in two dimensions. Chem. Rev. 120, 6197-6246 (2020)

124. S.I. Azzam et al., Ten years of spasers and plasmonic nanolasers. Light Sci. Appl. 9, $90(2020)$

125. Y. LeCun, Y. Bengio, G. Hinton, Deep learning. Nature 521, 436-444 (2015)

126. S. Molesky et al., Inverse design in nanophotonics. Nat. Photon $\mathbf{1 2}$ 659-670 (2018)

127. K. Yao, R. Unni, Y. Zheng, Intelligent nanophotonics: merging photonics and artificial intelligence at the nanoscale. Nanophotonics 8, 339-366 (2019)

128. S. So, T. Badloe, J. Noh, J. Bravo-Abad, J. Rho, Deep learning enabled inverse design in nanophotonics. Nanophotonics 9, 1041-1057 (2020)

129. W. Ma et al., Deep learning for the design of photonic structures. Nat. Photon. 15, 1-14 (2020)

130. N.H. Farhat, D. Psaltis, A. Prata, E. Paek, Optical implementation of the Hopfield model. Appl. Opt. 24, 1469-1475 (1985) 
131. D. Psaltis, N. Farhat, Optical information processing based on an associative-memory model of neural nets with thresholding and feedback. Opt. Lett. 10, 98-100 (1985)

132. Y. Shen et al., Deep learning with coherent nanophotonic circuits. Nat. Photon. 11, 441-446 (2017)

133. T.W. Hughes, M. Minkov, Y. Shi, S. Fan, Training of photonic neural networks through in situ backpropagation and gradient measurement. Optica 5, 864-871 (2018)

134. X. Lin et al., All-optical machine learning using diffractive deep neural networks. Science 361, 1004-1008 (2018)

135. J. Feldmann, N. Youngblood, C.D. Wright, H. Bhaskaran, W.H.P. Pernice, All-optical spiking neurosynaptic networks with self-learning capabilities. Nature 569, 208-214 (2019)

136. L. Mennel et al., Ultrafast machine vision with $2 \mathrm{D}$ material neural network image sensors. Nature 579, 62-66 (2020)

137. N. Mohammadi Estakhri, B. Edwards, N. Engheta, Inverse-designed metastructures that solve equations. Science 363, 1333-1338 (2019)

138. L. Li et al., Machine-learning reprogrammable metasurface imager. Nat. Commun. 10, 1082 (2019)

139. L. Xu et al., Enhanced light-matter interactions in dielectric nanostructures via machine-learning approach. Adv. Photon. 2, 026003 (2020)

140. Q. Zhang, H. Yu, M. Barbiero, B. Wang, M. Gu, Artificial neural networks enabled by nanophotonics. Light. Sci. Appl. 8, 42 (2019)

141. S. Zhang, Al empowered metasurfaces. Light. Sci. Appl. 9, 94 (2020)

142. J. Zhou, B. Huang, Z. Yan, J.G. Bunzli, Emerging role of machine learning in light-matter interaction. Light. Sci. Appl. 8, 84 (2019)

143. L. Su et al., Nanophotonic inverse design with SPINS: software architecture and practical considerations. Appl. Phys. Rev. 7, 011407 (2020)

144. K.Y. Yang et al., Inverse-designed non-reciprocal pulse router for chipbased LiDAR. Nat. Photon. 14, 369-374 (2020)

145. N.V. Sapra et al., On-chip integrated laser-driven particle accelerator. Science $\mathbf{3 6 7}, 79-83$ (2020)

146. Editorial. Deep learning gets scope time. Nat. Methods. 16, 1195 (2019).

147. E. Moen et al., Deep learning for cellular image analysis. Nat. Methods 16, 1233-1246 (2019)

148. C. Qian et al., Deep-learning-enabled self-adaptive microwave cloak without human intervention. Nat. Photon. 14, 383-390 (2020)

149. M.C. Rechtsman et al., Topological protection of photonic path entanglement. Optica 3, 925-930 (2016)

150. S. Mittal, V.V. Orre, M. Hafezi, Topologically robust transport of entangled photons in a 2D photonic system. Opt. Express 24, 15631-15641 (2016)
151. S. Barik et al., A topological quantum optics interface. Science 359, 666-668 (2018)

152. A. Blanco-Redondo, B. Bell, D. Oren, B.J. Eggleton, M. Segev, Topological protection of biphoton states. Science 362, 568-571 (2018)

153. M. Wang et al., Topologically protected entangled photonic states. Nanophotonics 8, 1327-1335 (2019)

154. N.A. Mortensen, S.I. Bozhevolnyi, A. Alù, Topological nanophotonics. Nanophotonics 8, 1315-1317 (2019)

155. M.S. Rider et al., A perspective on topological nanophotonics: current status and future challenges. J. Appl. Phys. 125, 120901 (2019)

156. M.I. Shalaev, W. Walasik, A. Tsukernik, Y. Xu, N.M. Litchinitser, Robust topologically protected transport in photonic crystals at telecommunication wavelengths. Nat. Nanotechnol. 14, 31-34 (2019)

157. H. Zhao et al., Non-Hermitian topological light steering. Science $\mathbf{3 6 5}$, 1163-1166 (2019)

158. S. Weidemann et al., Topological funneling of light. Science $\mathbf{3 6 8}$, 311-314 (2020)

159. L. Pilozzi, F.A. Farrelly, G. Marcucci, C. Conti, Machine learning inverse problem for topological photonics. Commun. Phys. 1, 57 (2018)

160. Y. Long, J. Ren, H. Chen, Unsupervised manifold clustering of topological phononics. Phys. Rev. Lett. 124, 185501 (2020)

161. E. Lustig, O. Yair, R. Talmon, M. Segev, Identifying topological phase transitions in experiments using manifold learning. Phys. Rev. Lett. 125 127401 (2020)

162. A.M. Palmieri et al., Experimental neural network enhanced quantum tomography. Npj. Quant. Inf. 6, 20 (2020)

163. W. Bogaerts, D. Pérez, J. Capmany et al., Programmable photonic circuits. Nature 586, 207-216 (2020)

164. X. Xu, M. Tan, B. Corcoran et al., 11 TOPS photonic convolutional accelerator for optical neural networks. Nature 589, 44-51 (2021)

165. G. Wetzstein, A. Ozcan, S. Gigan et al., Inference in artificial intelligence with deep optics and photonics. Nature 588, 39-47 (2020)

166. S. Stutzer et al., Photonic topological Anderson insulators. Nature $\mathbf{5 6 0}$ 461-465 (2018)

167. Z.A. Kudyshev, V.M. Shalaev, A. Boltasseva, Machine learning for integrated quantum photonics. ACS Photonics 8, 34-46 (2021)

168. K. Kawabata, K. Shiozaki, M. Ueda, M. Sato, Symmetry and topology in non-Hermitian physics. Phys. Rev. X 9, 041015 (2019)

169. S. Xia, et al. Nonlinear control of PT-symmetry and non-Hermitian topological states. Science $\mathbf{3 7 2}, 72-76$ (2021) 